\title{
Rauchen macht Brustkrebs aggressiver
}

Frauen, die Rauchen, haben im Hinblick auf eine Krebserkrankung eine deutlich schlechtere Prognose als Nichtraucherinnen. Das zeigen Daten der Collaborative Breast Cancer and Women's Longevity Study (CBCS). Bei den CBCS-Teilnehmerinnen waren 20.691 an einem lokalisierten oder regional invasivem Mammakarzinom erkrankt (Alter von 20 - 79 Jahre). Im Beobachtungszeitraum von median zwölf Jahren starben 6.778, darunter 2.894 brustkrebsbedingt.

Frauen, die bis zur Diagnose geraucht hatten, zeigten ein um $25 \%$ und damit signifikant höheres Risiko, an dem Brustkrebs zu sterben, als Nieraucherinnnen. Das höchste relative Risiko hatten Frauen mit einer Rauchbilanz von mindestens 30 Jahren bzw. 30 Packungsjahren. Keine erhöhte Brustkrebssterblichkeit hatte dagegen die Gesamtgruppe der Exraucherin- nen (Rauchstopp > 1 Jahr vor Diagnose). Nur wenn die ehemaligen Raucherinnen weniger als fünf Jahre vor der Diagnose aufgehört hatten, lag die brustkrebsbedingte Sterberate noch über der von tabakabstinenten Frauen (+20\%). Zudem starben Noch- und Exraucherinnen häufiger an Erkrankungen der Atemwege und des Herzkreislaufsystems; insgesamt lag ihre Mortalität um $11 \%$ bzw. $67 \%$ höher als die von Nieraucherinnen.

4.562 Patientinnen aus der CBCS-Kohorte wurden sechs Jahre nach der Diagnose erneut kontaktiert; von ihnen hatten $434(10 \%)$ das Rauchen nicht aufgegeben. Ihr Risiko, im medianen Follow-up von elf Jahren an Brustkrebs zu sterben, lag um $72 \%$ über dem von Frauen, die nie geraucht hatten. Im Gegensatz dazu hatten Frauen, die mit der Krebsdiagnose das Rauchen eingestellt hatten, eine nur noch

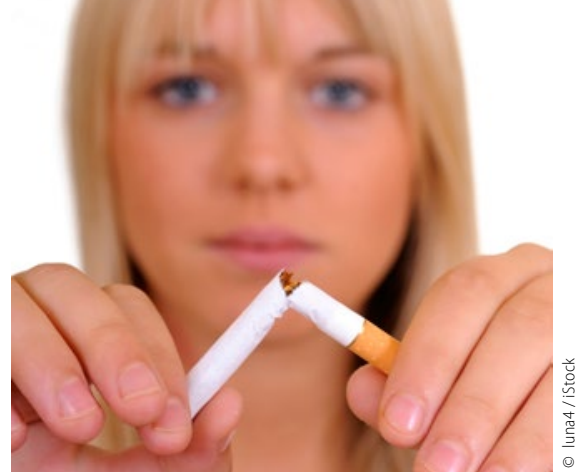

Nach der Diagnose Brustkrebs ist es höchste Zeit, ans Aufhören zu denken.

zahlenmäßig (+ 15\%), aber nicht mehr signifikant höhere Brustkrebsmortalität als Nieraucherinnen. Verglichen mit Frauen, die nach der Diagnose noch rauchten, war ihr Risiko für Brustkrebstod oder Tod im Studienzeitraum geringer $(-33 \%$ bzw. $-9 \%$ ), aber auch diese Unterschiede waren nicht signifikant. Signifikant seltener waren Todesfälle durch Krebserkrankungen der Atemwege oder des Herz-KreislaufSystems.

Beate Schumacher

Passarelli MN et al. Cigarette Smoking Before and After Breast Cancer Diagnosis: Mortality From Breast Cancer and Smoking-Related Diseases. J Clin Oncol 2016;34(12):1315-22.

\section{kurz notiert}

\section{Ausgezeichnet mit dem Walter-Trummert-Preis}

Der Verband der Medizin- und Wissenschaftsjournalisten e.V. (VMWJ) verleiht seit 1974 den Walter-Trummert-Preis an verdiente Medizinjournalisten. In diesem Jahr geht der Preis an Friederike Klein, freie Journalistin aus München. Nach dem Studium der Biologie begann Friederike Klein ihre berufliche Karriere 1990 als Lektorin.

Sie gelangte über verschiedene Stationen zum Verlag Urban \& Vogel, wo sie u. a. sechs Jahre lang den Bereich der Facharzt- und Pflegezeitschriften leitete. Zuvor hatte sie bereits die wissenschaftlichen Zeitschriften des Verlags in ihrer Obhut. In diese Zeit fällt auch die Gründung von "InFo Onkologie", die Friederike Klein gemeinsam mit Professor Ullrich R. Kleeberg auf ihren erfolgreichen Weg gebracht hat.

Seit 2005 ist Friederike Klein als freie Medizinjournalistin tätig, nach wie vor auch für das Fachgebiet Onkologie.

Friederike Klein beherrscht als Journalistin ein außerordentlich breites Themenspektrum. Zu ihren besonderen Kompetenzen gehört es, Veranstaltungen zu moderieren und den journalistischen Nachwuchs auszubilden. Der Vorstand des VMWJ hat sich einstimmig entschieden, Friederike Klein auszuzeichnen und damit die große Vielfalt sowie die hohe Qualität ihrer Arbeit zu würdigen.

Non-Hodgkin-Lymphome

\section{Etwas vereinfacht ausgedrückt: dick + groß = erhöhtes Lymphomrisiko}

Nach der Auswertung einer großen israelischen Studie ist das Risiko von sehr dicken und sehr großen Menschen an einem Non-Hodgkin-Lymphom (NHL) zu erkranken, um bis zu einem Viertel höher. Für die Studie hatte die Arbeitsgruppe um Merav Leiba die Aufzeichnungen zu mehr als 2,3 Millionen Israelis ausgewertet, die im Alter zwischen 16 und 19 Jahren gemustert worden waren. Das Alter bei der Musterung betrug im Schnitt 17 Jahre, $60 \%$ waren Männer. Zwischen Musterung und dem Ende des Analysezeitraums lagen im Mittel 20 Jahre (rund 48 Millionen Personenjahre). In dieser Zeit wurden 4.021 NHL diagnostiziert. Dabei erkrankten Männer um 25\% häufiger als Frauen. Bei Übergewichtigen und Adipösen war die NHL-Rate ebenfalls um $25 \%$ erhöht; pro $5 \mathrm{~kg}$ zu viel an Gewicht ließ sich eine Risikozunahme um $16 \%$ berechnen.

Ein deutliche, lineare Korrelation ergab sich auch mit der Körpergröße: Bei kleinen Menschen (unterhalb der 5. Perzenti- le) war die NHL-Rate verglichen mit der 25.-50. Perzentile um etwa $25 \%$ verringert, bei großen (über der 95. Perzentile) um etwa $25 \%$ erhöht. Übergewichtige und adipöse Personen hatten eine um $31 \%$ höhere Rate an diffusen großzelligen B-Zell-Lymphomen (DLBCL). Beim primär-kutanen Lymphom wr die Rate um $44 \%$ höher und beim Marginalzellenlymphom um $70 \%$.

Die größten Personen erkrankten 3-fach häufiger als die kleinsten an einem primär-kutanen Lymphom und 2,2-fach häufiger an einem DLBCL. Für follikuläre Lymphome, die chronisch lymphozytische Leukämie und das kleinzellige lymphozytische Lymphom ließen sich keine signifikanten Bezüge zu Größe und BMI herstellen.

Thomas Müller

Leiba M et al. Adolescent Weight and Height Are Predictors of Specific Non-Hodgkin Lymphoma Subtypes Among a Cohort of 2,352,988 Individuals Aged 16 to 19 Years. Cancer 2016;122(7):1068-77. 